\title{
Extraction of cellulose from waste papers for the preparation of cellulose graft-poly (acrylamide) for superabsorbent
}

\author{
Chamylle Rose Aquino ${ }^{1}$, Fauline Deune Carlos $^{1}$, Francis Padua $^{1}$, Honeylet Tayactac ${ }^{1}$, Adrienne Hera Zulueta ${ }^{1}$, Erison \\ Roque $^{1 *}$, and Nuna Almanzor ${ }^{2}$
}

${ }^{1}$ Department of Chemical Engineering, College of Engineering, Adamson University, 900 San Marcelino St., Ermita, Manila 1000, Philippines

${ }^{2}$ Center for Research and Development, Adamson University, 900 San Marcelino St., Ermita, Manila 1000, Philippines

\begin{abstract}
This article reports the use of wastepaper pulp as a direct source of cellulose for producing superabsorbent polymer. The white papers obtained from the offices in Adamson University were cut into 1 inch square pieces and drenched with distilled water and borax. This procedure was done couple of times to achieve a $\mathrm{pH}$ of 8 and consistency of $1 \%-2 \%$. Commercial cotton was used as control to compare and differentiate the properties and structures with the superabsorbent polymer (SAP). Various trials have shown the value of 60 acrylamide beads to produce SAP with optimum performance. FTIR analysis shows properties that are characteristics of cellulose-based SAP. In the present investigation, the influence of temperature, effect in varying salt concentrations, swelling rate and water retention at constant temperature were evaluated. The SAP's maximum swelling capacity ranges from $0.6561 \mathrm{~g}$ to $1.0638 \mathrm{~g}$ regardless of the amount of $\mathrm{NaCl}$ used. The swelling rate of the SAP proves that it gets heavier with time. Hence, it can be implied that the ability of the SAP to retain water is good. The temperature dependence data revealed the sorption process was favorable at higher temperature. The SAP will absorb the highest amount of solution at $500 \mathrm{C}$ given that the solution is pure.
\end{abstract}

\section{Introduction}

Superabsorbent polymers (SAP) are materials that can absorb a large amount of water by expanding and retaining the absorbed volume of water. It can hold large amount of water even if it is under pressure. This unique ability is the reason why it is most widely used in products used for personal hygiene such as diapers and sanitary napkins, and in medical applications as absorbent dressings. It has also found its way into other applications such as construction [1] and agriculture [2]. Majority of SAP applications, however, are disposable and $90 \%$ of SAPs used are synthetic and poor in degradability [3]. Because of this, there has been an interest in developing biopolymer based SAPs which are biodegradable [4]- [10]. Most of these studies have used plant matter as a source for biopolymers such as starch and cellulose.

Today, paper is a widely used material to make newspapers, books, magazines and such. Due to its wide use, the production of paper is projected to increase to 490 million tons by 2020 [11]. This increase in production results an increase in waste and involves waste disposal problems. The usual and most practical disposal method is to use a landfill but it has a consequence of periodic fires, contamination of water sources and a possible breeding ground for numerous diseases. Incineration is another way of disposing materials but this process tends to produce lots of pollution. SAP can be used to increase the moisture of the soil and as a result decrease the times of irrigation. In this study, paper wastes were used as a source of the cellulose biopolymer for SAP. The characteristics of SAP was analyzed using FTIR for the functional groups and SEM for the surface morphology. The maximum swelling capacity was determined at different $\mathrm{NaCl}$ concentrations. Also the effect of absorption time, water retention and temperature on the swelling capacity of SAP evaluated.

\section{Materials and methods}

\subsection{Materials and reagents}

White waste papers were acquired from the different offices of a university. Borax and hydrochloric acid were purchased at Puljed Trading Corporation while the acetyl acetone and acrylamide used were from Adamson University Testing Research Development Center (AUTRDC).

\subsection{Pulping and deinking}

The wastepaper was cut into pieces that are 1 square

* Corresponding author: erison.roque@ adamson.edu.ph 
inch in size or smaller and then drenched in distilled water added with borax to achieve a $\mathrm{pH}$ of 8 for 12 hours at room temperature. The mixed waste paper was filtered and then mixed again with distilled water and borax to achieve a $\mathrm{pH}$ of 8 and a consistency of $1-2 \%$. The mixture was fed to a blender for five minutes to produce fine pulp. The fine pulp was filtered and deinked and washed with detergent. This process was repeated until the filtrate was no longer gray. The pulp was rinsed with distilled water, filtered and pressed with a cheese cloth. This process was repeated until the filtrate has no more suds. The pulp was bleached to further remove smaller ink residues. Afterwards, the pulp was filtered and pressed several times using a cheese cloth.

\subsection{Preparation of superabsorbent polymers}

A $2 \mathrm{~g}$ of commercially available cotton and cellulose and a 30, 45 and 60 pieces of acrylamide was blended with $500 \mathrm{ml}$ of water. After blending, $5 \mathrm{ml}$ of acetylacetone was added to the mixture. This mixture was heated for $30 \mathrm{~min}$ and filtered with a thin cloth. The water content of the product was determined.

\subsection{Analytical and test method}

To measure the swelling capacity $\left(\mathrm{S}_{\mathrm{e}}\right), 2$ grams of superabsorbent sample $\left(\mathrm{W}_{0}\right)$ was placed in a tea-bag and immersed in a $750 \mathrm{~mL}$ of $0.50 \%$ to $0.70 \% \mathrm{NaCl}$ for an hour to achieve the swelling equilibrium (W1). The bags were hanged to remove the extra solution and then weighed again until there are no more liquids coming off.

The swelling kinetics of the hydrogel was determined by preparing $2 \mathrm{~L}$ of $0.50-0.70 \% \mathrm{NaCl}$ solution. The SAP was placed in a flask and gently stirred. The swelling rate of the superabsorbent polymer was tested at $5 \mathrm{~min}, 10 \mathrm{~min}$ and 20 minutes swelling time.

At room temperature, the superabsorbent polymer was immersed in distilled water until the swelling equilibrium was reached. Using a weighed 100-mesh nylon bags, the equilibrated gels were drained. Nylon bags containing hydrogels were placed inside an oven and heated at $40{ }^{\circ} \mathrm{C}$. The weights of the bag were measured every 10, 20 and $30 \mathrm{~min}$ to relate their weight and heating time.

A batch process was done to examine the swelling properties of the hydrogel beads at equilibrium. The hydrogel beads with the same mass and diameter was placed in a $20 \mathrm{~mL}$ distilled water at $\mathrm{pH}$ of 7 . It was left for $10 \mathrm{~min}$ at a temperature of $30{ }^{\circ} \mathrm{C}$ to $50{ }^{\circ} \mathrm{C}$. Then, it was tested for $10 \mathrm{~min}$. It was also tested in a saline solution.

0.4 grams of SAP was covered by two layers of nylon cloth was embedded under the soil surface with 10 $\mathrm{cm}$ depth. Samples implanted in the $10 \mathrm{~cm}$ depth from the soil surface was placed in two temperature humidity chambers at $32{ }^{\circ} \mathrm{C}$ and $40{ }^{\circ} \mathrm{C}$ at an interval of 3 days for 3 times.

\section{Results and discussion}

\subsection{FTIR analysis}

The presence of hydroxyl $\left({ }^{-} \mathrm{OH}\right)$ was manifested by a strong, broad peak centered at $3298.02 \mathrm{~cm}^{-1}$ indicates that the compound of interest may contain the ${ }^{-} \mathrm{OH}$ groups of an alcohol functionality. The compound possibly only contains alcohol groups for there is absence of intensity, at $1700 \mathrm{~cm}^{-1}$ stretching frequency with carbonyl $(-\mathrm{C}=\mathrm{O})$ groups can be found. Between the stretching frequencies of the ${ }^{-} \mathrm{OH}$ group and $\mathrm{sp}^{2}$ hybridized, overlaps can be seen. Also, carbon can't be disregarded. A peak at $2971 \mathrm{~cm}^{-1}$ may be recognized to the stretching frequencies of a saturated ${ }^{-} \mathrm{C}-\mathrm{H}$ moiety. Two symmetrical sharp points around $1657.47 \mathrm{~cm}^{-1}$ and $1552.62 \mathrm{~cm}^{-1}$ may indicate the occurrence of the hybridized carbon chain of an aromatic group. Presence of these functional groups may indicate stretching of the

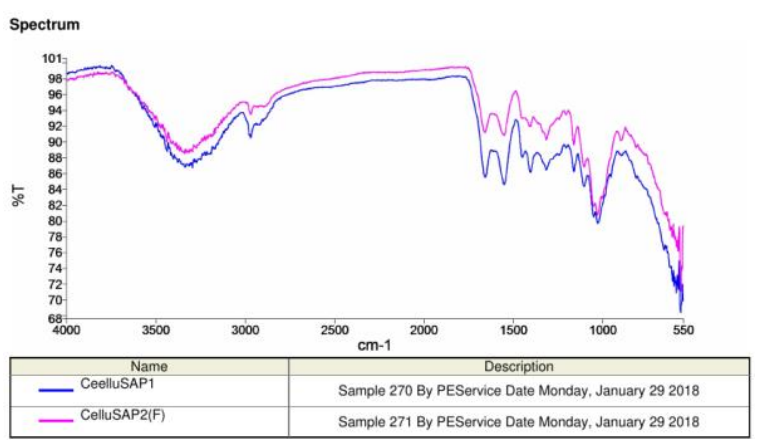

Figure 1. FTIR Analysis. SAP from cotton in blue and SAP from waste paper in pink

$\mathrm{sp}^{2}$ that the sample contains molecules with an alcohol and ether moiety possibly, cellulose. The stretching vibrations of the hydrogen bonded hydroxyl group has a broad peak centered at $3341.28 \mathrm{~cm}^{-1}$. However, possible overlap of the peaks associated for the stretching frequencies for $\mathrm{sp}^{2}$ hybridized carbons of an alkene group or an aryl group cannot be dismissed. Peaks at approximately $2900 \mathrm{~cm}^{-1}$ are usually for the alkenyl stretch of $-\mathrm{C}-\mathrm{H}$ groups within the molecule. The inplane bending vibrations of the $\mathrm{C}-\mathrm{C}$ chain of an aromatic group resulted a doublet splitting pattern seen at peaks $1657.17 \mathrm{~cm}^{-1}$ and $1551.64 \mathrm{~cm}^{-1}$ Medium to strong, sharp peaks found at the fingerprint region may be indicative of the presence of an ether group. Presence of these functional groups indicate that the sample contains molecules with an alcohol and ether moiety possibly, cellulose. However, other instrumental methods of analysis are pertinent in order to conclusively define the compound.

\subsection{SEM Analysis}

From the surface images shown in Figures 2-5, it can be inferred that the structures of SAP and cotton are almost the same. The cellulose produced were taken at 1000 times magnification (Figure 2) and at 500 times magnification (Figure 3). Whereas, the surface 


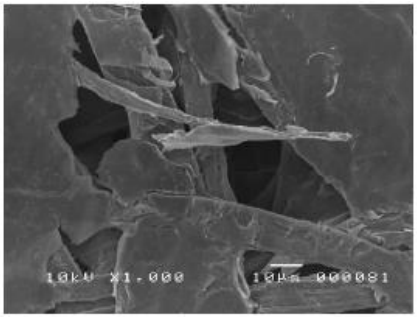

Figure 2. Cellulose at $\times 1000$ Magnification

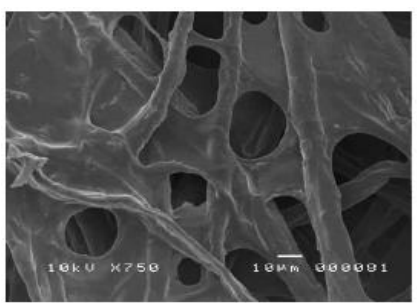

Figure 2. Cotton at $\times 75$ Magnification

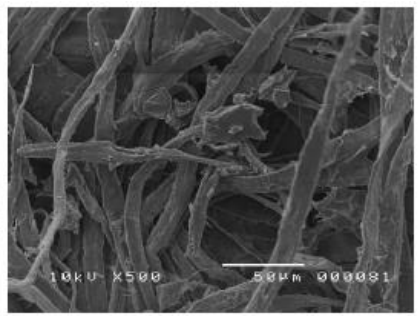

Figure 3. Cellulose at $x 500$ Magnification

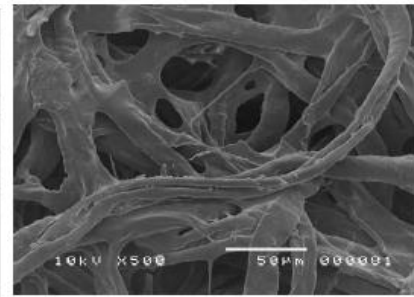

Figure 5. Cotton at $\times 500$ Magnification morphology of the cotton was taken at 700 times magnification (Figure 4) and 500 times magnification (Figure 5). The images showed the same morphological structure.

\subsection{Swelling capacity}

It can be seen from Figure 6 that the SAP can swell from 0.6561 to 1.0638 regardless of the amount of $\mathrm{NaCl}$ used. Also on the average, the weight of the SAP is equal to 0.8285 . Looking at the sub analysis, when there is $0.50 \%$ $\mathrm{NaCl}$ mixed, the SAP ranges from 0.8270 to 0.8897 and weighs 0.8591 on the average. On the other hand, SAP weighs 0.7651 to 0.7809 when there is $0.60 \% \mathrm{NaCl}$ present and is typically equal to 0.7712 . It weighs 0.6561 to 0.6772 when the amount of $\mathrm{NaCl}$ present is $.70 \%$ and 0.6656 on the average. When there is no $\mathrm{NaCl}$ present, the range of the weight of SAP is from 0.9796 to 1.0638 and its average weight is 1.0179 . Thus, the SAP is able to absorb the highest amount of pure solution while the least amount it absorbs is the solution with $0.70 \% \mathrm{NaCl}$.

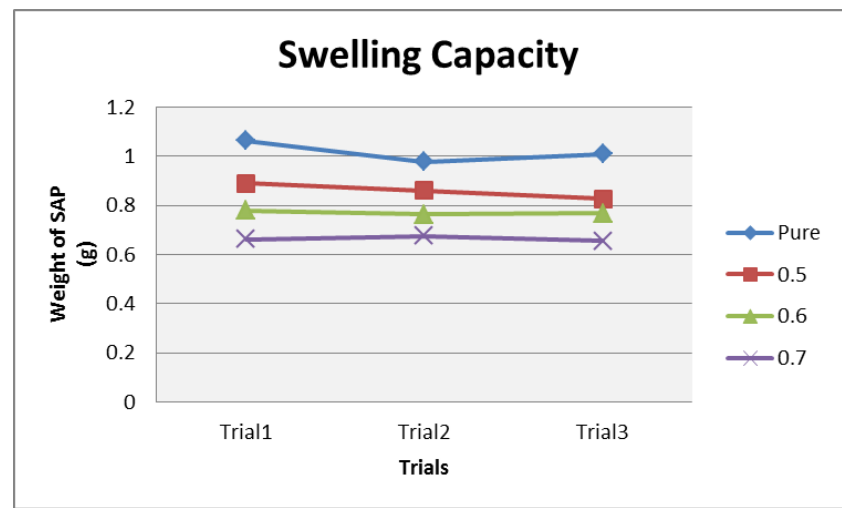

Figure 6. Swelling Capacity

The data gathered for this test were treated using Jonckheere-Terpstra Test for Ordered Alternatives and Wilcoxon Mann-Whitney Test methods in order to determine how well the data fits the model and which parameters considered in this research were actually significant. In this case, the $\mathrm{R}^{2}$ is equal to 0.1368 . At $\mathrm{p}$ value $\mathrm{p}<0.05$ level, the model obtained a $\mathrm{p}$-value of less than 0.0001 considering it to be significant. In comparison between SAP and cotton, it gathered a pvalue of 0.069 which is greater than 0.05 that makes it inconsiderable. There is no sufficient evidence to conclude that there is significant difference between final and initial weight of the SAP and commercially available cotton.

There is sufficient evidence to conclude that the larger the amount of $\mathrm{NaCl}$ present, the smaller the amount of water absorbed by the SAP. Figure 6 supports the result of the hypothesis test where there is sufficient evidence to conclude that SAP absorbed large amount compared to the salt solution and it varies inversely with its concentration. As the salt concentration gets higher, the ability of SAP absorption decreases.

\subsection{Effect of time during absorption}

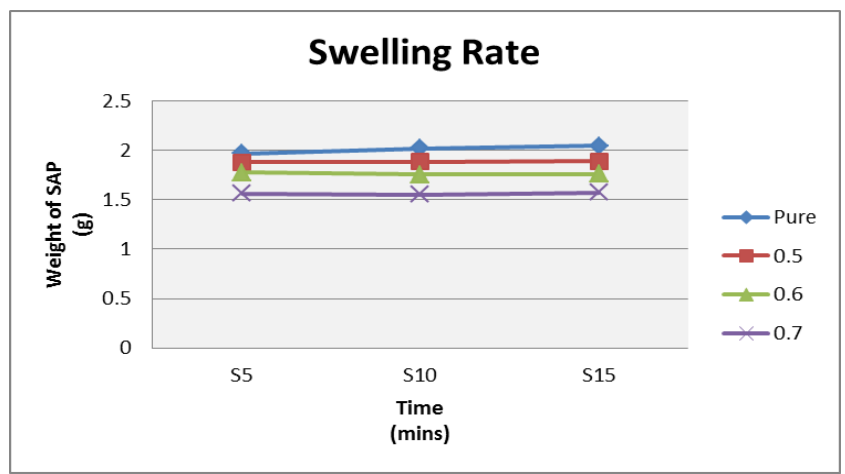

Figure 7. Swelling rate

Figure 7 shows us that SAPs that were removed from the solution are weighed after 5 minutes and that is 1.7972 on the average and is at least 1.4957 to 2.0008 . The weight of the SAP after 10 minutes averages 1.8041 and ranges from 1.5013 to 2.1096 while it is commonly 1.8179 and is at least 1.5097 to 2.1369 when weighed after 15 minutes. It also shows the ranking of the three different time points and it was proven that the SAPs are lightest when it is weighed just after 5 minutes and is heaviest when weighed after 15 minutes, multiple comparisons were done. It is proven that the SAP gets heavier with time. The difference is mainly seen when it is weighed after 10 minutes and after 15 minutes. Significant difference, however, from its weight after 5 minutes and after 10 minutes cannot be seen since it is very small or is almost negligible. The figure shows that as time passes the SAP is able to absorb more solution.

\subsection{Water retention at constant temperature}

It can be seen from Figure 8 that the weight of the SAP after 10, 20 and 30 minutes is decreasing. In each of these time periods, the maximum values were all 2.18 and starts at $1.65,1.63$, and 1.59 respectively. It can be seen from the output above the difference between the weights of the water removed from the SAP when 
weighed after 10 minutes and when it is weighed after 20 minutes ranges from 0.0015 to 0.0075 . On the average, their differences are just equal to 0.0045 .

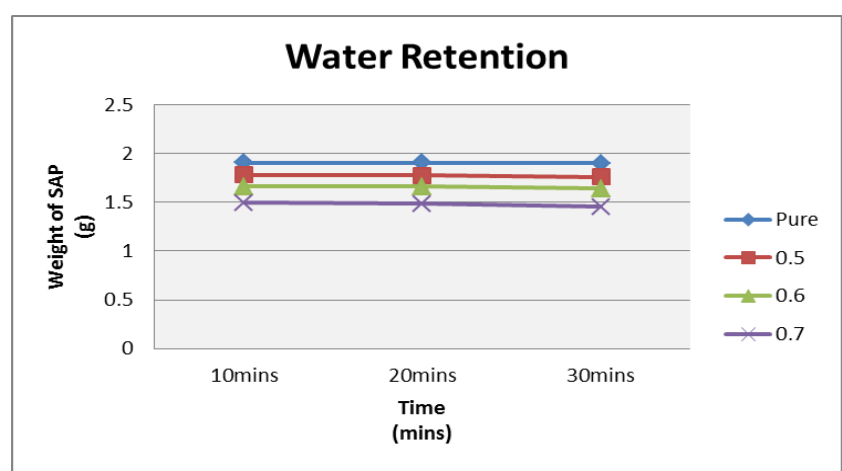

Figure 8. Effect of time in water retention

The differences of weights from these time points are significantly very small. However, the difference of the weights of the water removed from the SAP after 20 and 30 minutes is, at least, 0.0060 and at most, 0.0321 . Its average value is 0.0190 , which is slightly larger than that of the average difference of the 10 and 20 minute weights. The difference, therefore, is evidently bigger between 20 and 30 minutes than the difference of weights at 10 and 20 minutes.

Figure 8 also shows the ranking of the three different time points. Since it is proven that the SAPs still retained a lot of water, though there is a significant value of water removed after 30 minutes and just after 10 minutes, the value of water removed is negligible.

Although the result of the hypothesis test concludes that the SAP gets lighter with time, it can be observed in the graph that the weight of the SAP after 30 minutes does not deviate much from its weight in 10 minutes. Hence, it can be implied that the ability of the SAP to retain water is good.

\subsection{Temperature of the solution being absorbed}

It can be seen from Figure 9 that at $30{ }^{\circ} \mathrm{C}$, the weight of the SAP ranges from 0.6991 to 1.1093 and is 0.9236 on average. At $40{ }^{\circ} \mathrm{C}$, it is at least 1.0021 to 1.1301 and has an average of 1.0765 . At $50{ }^{\circ} \mathrm{C}$, the weight of the SAP is usually 1.1354 and is around 1.0290 to $1.5880 \mathrm{~g}$.

Figure 9 supports the result of the test where there is sufficient evidence to conclude that the superabsorbent gets heavier as the temperature gets higher. At $50{ }^{\circ} \mathrm{C}$, the SAP was able to absorb a high amount of $0.70 \% \mathrm{NaCl}$ solution, while at $30{ }^{\circ} \mathrm{C}$, only a small amount of solution was absorbed by the SAP. Given that the solution is pure, the SAP will absorb the highest amount of solution at $50{ }^{\circ} \mathrm{C}$.

\section{Effect of Temperature of the Solution in the Absorbency}

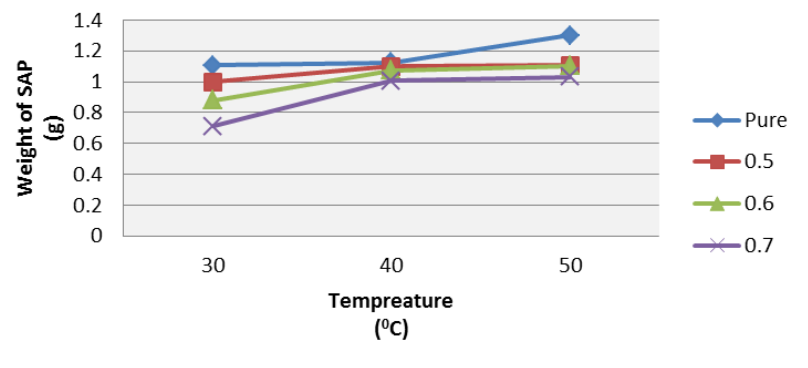

Figure 9. Effect of Temperature on Retention weight

\section{Conclusion}

A superabsorbent material from waste paper and was able to compare it with the superabsorbent from cotton made with the same exact procedure. There is sufficient evidence to conclude that, within a saline solution, the swelling capacity of the material is inversely proportional to the salt concentration. It was also shown that, within a solution, the SAP gets heavier with time, however, there wasn't a significant difference between the weight after 5 minutes and after 10 minutes compared to 10 minutes and 15 minutes. The superabsorbent polymer is able to retain water up to 30 minutes without a significant difference from the initial swelled SAP. The SAP is also shown to be affected by effects of temperature to the swelling capacity and that, as the temperature of the solution increases, the swelling capacity also increases. It is then recommended for the future researchers for this topic to try to increase the number of commercially available cotton to use in order to compare it with the SAP.

The researchers would like to extend gratitude to Center of Research and Development of Adamson University for making this research possible.

\section{References}

[1] Senff, L., Modolo, R. C. E., Ascensão, G., Hotza, D., Ferreira, V. M., \& Labrincha, J. A. (2015). Development of mortars containing superabsorbent polymer. Construction and Building Materials, 95, 575-584.

[2] Gao, L., Wang, S., \& Zhao, X. (2013). Synthesis and characterization of agricultural controllable humic acid superabsorbent. Journal of Environmental Sciences, 25(S1), S69-S76.

[3] Liu, Z., Miao, Y., Wang, Z., \& Yin, G. (2009). Synthesis and characterization of a novel superabsorbent based on chemically modified pulverized wheat straw and acrylic acid. Carbohydrate Polymers, 77(1), 131-135.

[4] Feng, H., Li, J., \& Wang, L. (2010). Preparation of biodegradable flax shive cellulose-based superabsorbent polymer under microwave irradiation. BioResources, 5(3), 1484-1495. 
[5] Li, A., Zhang, J., \& Wang, A. (2007). Utilization of starch and clay for the preparation of superabsorbent composite. Bioresource Technology, 98(2), 327-332.

[6] Li, J., Ji, J., Xia, J., \& Li, B. (2012). Preparation of konjac glucomannan-based superabsorbent polymers by frontal polymerization. Carbohydrate Polymers, 87(1), 757-763.

[7] Li, Q., Ma, Z., Yue, Q., Gao, B., Li, W., \& Xu, $X$. (2012). Synthesis, characterization and swelling behavior of superabsorbent wheat straw graft copolymers. Bioresource Technology, 118, 204-209.

[8] Ma, Z., Li, Q., Yue, Q., Gao, B., Xu, X., \& Zhong, Q. (2011). Synthesis and characterization of a novel super-absorbent based on wheat straw. Bioresource Technology, 102(3), 2853-2858.

[9] Sawut, A., Yimit, M., Sun, W., \& Nurulla, I. (2014). Photopolymerisation and characterization of maleylatedcellulose-gpoly(acrylic acid) superabsorbent polymer. Carbohydrate Polymers, 101(1), 231-239.

[10]Zhang, J., Wang, L., \& Wang, A. (2007). Preparation and Properties of Chitosan- g poly(acrylic acid)/Montmorillonite Superabsorbent Nanocomposite via in Situ Intercalative Polymerization. Industrial \& Engineering Chemistry Research, 46(8), 24972502.

[11] Bajpai, P. (2014). Water Reuse, Wastewater Treatment and Closed-Cycle Operation. In Recycling and Deinking of Recovered Paper (pp. 251-269). 